\title{
Diversidad y cohesión: arte, ecosistemas, sociedades
}

\author{
Diversity and cohesion: art, ecosystems, societies
}

Resumen

En este texto se exploran la diversidad y la cohesión como dos variables en tensión, importantes para los ecosistemas, la creación artística y las sociedades. Se propone, a partir de la filosofía de Bateson y la de Spinoza, que es posible y deseable tener alta variedad y alta cohesión al mismo tiempo. El documento destaca evidencias de que éstas son condiciones necesariamente implicadas en los procesos de evolución y de desarrollo propios de todo sistema, que tienden a ser cada vez más complejos. La complejidad es inherente a la sostenibilidad de los sistemas biológicos y sociales, por lo que se sugiere entonces que la riqueza y el sostenimiento de la vida dependen de la diversidad y la cohesión, haciendo posible la prolongación de la creación y la adaptación a nuevas estructuras.

Palabras Clave: Filosofía de la biología, estética, política, Bateson, Spinoza, Whitman, diversidad, cohesión.

\begin{abstract}
This text explores diversity and cohesion as two variables in tension - important for ecosystems, artistic creation and societies. Following the philosophies of Bateson and Spinoza, it proposes that it is possible and desirable to have great variety and a high level of cohesion at the same time. The document stresses evidence that these are conditions necessarily implicated in the processes of evolution and development present in all systems, which tend to be increasingly complex. Complexity is inherent in the sustainability of biological and social systems, so it is suggested that wealth and the sustenance of life depend on diversity and cohesion, making possible the prolongation of creation and adaptation to new structures.
\end{abstract}

Keywords: Philosophy of biology, esthetics, politics, Bateson, Spinoza, Whitman, diversity, cohesion. 


\section{Diversidad, cohesión y co-evolución}

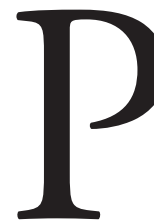

ensemos en un sándwich bien sencillo: dos tajadas de pan blanco y una de queso holandés. Sin duda se trata de un sándwich, si bien bastante aburrido. Añadamos una tajada de jamón, algo de mostaza y lechuga crespa. Acabamos de aumentar la diversidad del sándwich sin sacrificar su cohesión, esto es, su identidad como sándwich. Es más sabroso. Pongamos aceitunas verdes y negras, espinaca, pimienta, pepinos, miel, un guiso de pimentones y tomates, almendras, maíz tostado, cebollitas rojas en conserva, chiles, sardinas en aceite, etc. ¿En qué momento dañamos el sándwich? ¿Cuándo pasó de ser un plato progresivamente más rico en sabores y texturas a convertirse en un desastre culinario? En algún momento el sándwich perdió su identidad; una cosa unitaria se convirtió en un revuelto de ingredientes sin una trama común. He aquí una tensión entre dos variables, diversidad y cohesión. Una creación culinaria puede fracasar por carecer de una u otra, pero ambas están en cierto antagonismo mutuo. Bien, la culinaria es una habilidad, un arte que debe cultivarse.

¿Qué debe permitir una sociedad, qué debe prohibir? Siendo un acto consensual, la mayoría de las sociedades modernas — sociedades liberales - prohíben la poligamia. Si bien estas mismas sociedades profesan creer en las bondades del mercado libre, reservan a los bancos centrales el monopolio de la emisión de dinero. Mientras más cohesión tenga una sociedad más sólida será, además de resistente al cambio, a la erosión y a la destrucción. Mientras más diversidad tenga, más rica será la vida y mayores las posibilidades de transformación y crecimiento.

¿Proponemos una doctrina del justo medio? ¿Se trataría entonces de encontrar el punto máximo de diversidad después del cual comienza a perderse la cohesión? No es necesario conformarse con esto. Aquí se propone que, si bien las dos variables están en tensión, es posible maximizar ambas, a través de un manejo hábil. Como evidencia, citamos dos ejemplos: las sinfonías de J. S. Bach y la "gran sinfonía de la naturaleza".
¿Por qué acudimos a Bach? No sabemos de otro compositor que haya sido tan hábil para integrar múltiples y diversas ideas musicales en una sola composición. El clavecín bien temperado, por ejemplo, se considera una obra maestra del contrapunto (es decir, poner a dialogar varias líneas melódicas), en la que ninguna fuga se parece, y no obstante conforman una unidad con coherencia estética. Quien escucha la música puede, alternativamente, concentrarse en escuchar el todo; o bien distinguir un par de líneas melódicas y deleitarse en su juego mutuo; o bien comenzar mentalmente por un instrumento, e ir ańadiendo de uno a uno para apreciar cómo todos hacen parte de una sola sinfonía, al tiempo que son diferentes. He aquí una obra que logra la diversidad sin sacrificar la cohesión, o viceversa. ¿De qué manera? Por el momento, diremos que Bach fue un músico excelente.

La diversidad y la cohesión coexisten también en un ecosistema maduro: una selva tropical, un sistema de manglares, un bosque andino. Allí, una gran variedad de especies (animales, vegetales, grandes y pequeñas), cada una enormemente compleja en sí misma, conviven en un equilibrio que constituye un sistema de orden superior.

Es fácil caer en una "romantización" de la naturaleza, en concordancia con algunos lugares comunes de nuestro tiempo: la naturaleza es buena, el hombre es malo, hay un equilibrio en todo lo natural, etc. No pretendemos acoger esta visión romántica y sobre-simplificada; hablamos de propiedades concretas de los ecosistemas maduros, no de idealizaciones "antropomorfizantes". Cuando un lobo se come a un ciervo, esto es una mala noticia para el ciervo. Los lobos, que generalmente buscan especímenes enfermos, suelen atacar la pelvis o la garganta del ciervo, con mordiscos que producen perforaciones de 10 a $15 \mathrm{~cm}$ de longitud, con lo que un ciervo puede morir desangrado tras un par de mordiscos.

No obstante, el ecosistema al que pertenecen lobo y ciervo puede estar en equilibrio, y funcionar para el beneficio mutuo de las especies en cuestión. Lo importante es distinguir entre el ciervo individual y la población de ciervos en un determinado ecosistema. Cuando el lobo Pedro 
come al ciervo Juan, le hace un mal a Juan. No obstante, Pedro contribuye, junto con su especie, a hacerle dos favores a la especie de Juan, a mediano y a largo plazo. A mediano plazo, Pedro contribuye a controlar la población de ciervos; sin depredadores, éstos crecerían más allá de la capacidad del ecosistema para sostenerlos. A largo plazo, al reducir las probabilidades de reproducción de los especímenes poco adaptados, los lobos contribuyen a la evolución de los ciervos. A su vez, estos ciervos mejor adaptados serán malas noticias para individuos como Pedro, pero impulsarán a la raza de lobos hacia la evolución.

Es mediante un largo proceso de co-evolución que los ecosistemas maduros llegan a tener, al mismo tiempo, una alta variedad y una alta cohesión. Los cambios de una especie, que responden al entorno físico y a otras especies, determinan cambios en otras especies, ajustando de manera apretada las diferentes piezas de una totalidad determinada. Con el tiempo, se produce un ecosistema altamente acoplado y diverso, y por tanto resistente a perturbaciones -la multiplicidad de especies permite que el ecosistema persista aunque algunas de ellas sufran perturbaciones, pues hay otras que pueden suplir la función de las primeras-, en el que especies diferentes aprovechan todos los nichos que están disponibles. El naturalista Edward Wilson describe vívidamente su experiencia en la selva de Bernhardsdorp, Surinam:

[...] Se encontraban allí innumerables productos de la evolución, por propósitos que nada tenían que ver conmigo; su larga historia cenozoica está encriptada en un código genético que no podía entender. Producía un efecto extrañamente tranquilizante. Se redujeron la respiración y el ritmo cardiaco. Me pareció que algo extraordinario en el bosque estaba muy cerca de donde me encontraba, moviéndose hacia la superficie y el descubrimiento.

Me enfoqué en unos pocos centímetros de piso y vegetación. Me esforcé por hacer materializar a los animales, y entraron de manera errática en $\mathrm{mi}$ visión. Flotaban hacia abajo mosquitos azul metálico desde la canopea en busca de algo de piel descubierta, cucarachas con alas multiformes se posaban como mariposas sobre las hojas iluminadas por el sol, hormigas carpintero de color negro cubiertas de cabellos dorados marchaban con afán a través de un madero en putrefacción. Viré mi cabeza ligeramente y todo desapareció. Todo esto no era sino una fracción infinitesimal de la vida que allí estaba presente. La selva era una vorágine biológica que sólo mostraba su superficie al ojo desnudo. Dentro de mi círculo de visión, millones de organismos morían cada segundo desapercibidos. Su destrucción era rápida y silenciosa[...] Los cuerpos microscópicos eran descompuestos mediante nítidos cortes bioquímicos por parte de depredadores y carroñeros, y luego asimilados para crear millones de organismos nuevos, cada segundo (Wilson, 1984, p. 7). ${ }^{1}$

El resultado no se asemeja a un collage en el que coexisten elementos dispersos; la diversidad forma una sinfonía cohesionada e identificable. Visitar un ecosistema maduro debería ser, más que tomar fotos de esta o esta otra especie sobresaliente, un asunto de aprender a reconocer esta sinfonía. Por ejemplo, ver el juego entre las mareas y las diversas plantas y moluscos que crecen en un manglar, y el juego entre los moluscos y las aves marinas, y los peces que se alimentan en las raíces de los árboles. Una postal de un cangrejo azul es algo bonito, pero está en relación con entender un ecosistema como escuchar una sola nota está en relación con escuchar, y poder seguir, El clavecín bien temperado de Bach.

A diferencia de la obra de Bach, la complejidad de un ecosistema maduro sobrepasa ampliamente nuestra capacidad de entenderla a plenitud. Esto porque, en palabras de Leibniz,

[...] cada cuerpo orgánico es una especie de Máquina divina o de Autómata Natural, que sobrepasa infinitamente a todos los Autómatas artificiales. Porque una máquina hecha por el arte del hombre no es máquina en cada una de sus partes o fragmentos[...] Pero las Máquinas de la Naturaleza, es decir, los cuerpos vivos, son, sin embargo, Máquinas en sus menores partes hasta el infinito. Esto es lo que constituye la diferencia entre la Naturaleza y el Arte, es decir, entre el arte Divino y el Nuestro (Leibniz, 1983, \$64).

A la complejidad de la naturaleza, a un nivel determinado de observación, se suma toda

1 Todas las citas de Wilson traducidas del inglés al español por el autor. 
la complejidad a niveles superiores e inferiores de observación. Cada parte de cada nivel de conexión está conectada entre sí, y cada nivel de conexión con los otros:

Adentro [de la selva] era un mar viviente a través del cual me movía como un buzo a tientas[...] Pero sabía que a mí alrededor todas las piezas y partes, los organismos individuales y las poblaciones, trabajan con extrema precisión. Algunas de las especies estaban entrelazadas en formas de simbiosis tan intrincadas que remover una llevaría a la otra a la extinción. Tal es la consecuencia de la adaptación por co-evolución, el cambio genético recíproco de las especies que interactúan entre sí a través de muchos ciclos de vida. [...]

Después de que la energía del sol es capturada por las plantas verdes, fluye a través de cadenas de organismos de manera dendrítica, como sangre pasando de las arterias a las redes de capilares sanguíneos microscópicos. Es en estos capilares, en los ciclos de vida de miles de especies individuales, que se lleva a cabo el trabajo importante de la vida. Por tanto nada en el sistema total tiene sentido hasta que se conoce la historia natural de las especies constituyentes. El estudio de cada organismo es importante, en cada lugar del mundo[...] (Wilson, 1984, p. 8).

Mientras que, con cierto esfuerzo, podemos comprender todas las partes de la música de Bach en relación con el todo, de la sinfonía de la naturaleza apenas podemos aspirar a percibir algunas líneas melódicas, algunos contrapuntos fruto de la co-evolución:

El perezoso tridáctilo se alimenta de hojas de la canapea de las selvas bajas de grandes partes de América Central y del Sur. En su pelaje viven diminutas polillas, Cryptoses tholoepi, que no es encuentran en ningún otro lugar de la tierra. Cuando un perezoso desciende al suelo de la selva a defecar (una vez por semana), las polillas hembra dejan brevemente el pelaje para depositar sus huevos en las heces frescas. Las orugas que de ahí salen hacen nidos de seda y comienzan a alimentarse. A las tres semanas completan su desarrollo y se convierten en polillas adultas, y vuelan hasta la canapea en busca de perezosos. Al vivir directamente en los cuerpos de los perezosos, las Cryptoses adultas aseguran que sus descendientes tengan fácil acceso al excremento rico en nutrientes, dándoles una ventaja competitiva sobre todo una multitud de otros organismos coprófagos (Wilson, 1984, p. 8-9).
La naturaleza no sólo ofrece una complejidad infinita en términos asincrónicos. Lo que podemos ver en un momento determinado, es el resultado de una enorme historia evolutiva que añade una dimensión adicional de complejidad. Detrás de la polilla y el perezoso hay una serie de "proto-polillas" y "proto-perezosos" en entornos cambiantes, pasando por diversas e igualmente complejas fases de co-evolución. ¡Qué diferente es introducirse en esta complejidad a sacar fotos de algunos animales exóticos! ¡Cuánto nos hace falta aprender a disfrutar de la naturaleza!

Gregory Bateson, en su libro Espiritu y naturaleza (1993) ha mostrado que la manera en que Bach, por un lado, y un ecosistema maduro, por otro, son creativos, es la misma. La emergencia de lo nuevo requiere, si ha de ser nuevo, de la aleatoriedad, del azar. En el ecosistema, se trata de las mutaciones aleatorias, de los errores en la reproducción, de las diversas especies. En el arte, se trata del capricho, del vuelo de la imaginación.

Ahora bien, la verdadera creatividad no consiste tan sólo en la aleatoriedad, en el capricho (este malentendido es el que ha llevado al arte contemporáneo a considerar artística una mancha de orín en una pared, siempre y cuando el orín haya provenido de un artista reconocido). Además de la aleatoriedad hay un segundo elemento en la creatividad, un elemento conservador y riguroso sin el cual la aleatoriedad es mero capricho.

En los procesos evolutivos, la aleatoriedad es sometida a dos filtros que hacen que la aleatoriedad sea coherente con el orden pre-existente. Por un lado están los procesos ontogenéticos que garantizan que la diversidad introducida por las mutaciones aleatorias sea compatible con las estructuras ya existentes; es decir, los procesos en la formación del embrión que impiden mutaciones que comprometan la integridad del organismo. Por otro, sólo sobreviven las mutaciones que, puestas a jugar con su entorno, tienen un valor adaptativo (Bateson, 1993).

En el arte, la aleatoriedad también tiene filtros de coherencia interna y externa. Por un lado, toda novedad introducida por el vuelo de la imagi- 
nación, por lo aleatorio, debe hacerse coherente con la estructura misma de la obra; por ejemplo, el trabajo del contrapunto, mediante el cual se logra que diversas ideas musicales sean coherentes entre sí. Por otro, hay una coherencia externa de la obra con cánones artísticos pre-existentes: sea que la obra se ubique dentro de una tradición o que intente modificarla, escandalizarla o destruirla, siempre hay un diálogo entre la obra y su entorno artístico.

Vale la pena insistir en este punto, y aún introducir una distinción. Llamemos fantasía al paso aleatorio del proceso creativo, al vuelo de la imaginación que introduce novedad en la creación artística. Llamemos creatividad al proceso completo, en el que la fantasía es sometida al oficio y hecha coherente tanto con el interior de la obra a la que aporta como al entorno de la misma. La fantasía sin oficio no es más que ruido: el niño, el loco y el consumidor de LSD abundan en fantasía, pero no por ello son grandes artistas. Sólo el oficio, el conocimiento de los cánones con referencia a los cuales se trabaja, permite a los grandes artistas quebrarlos, crear nuevos cánones (que a su vez serán quebrados por otros artistas).

Así pues, Espíritu y Naturaleza no son dos sustancias opuestas, sino que comparten una misma manera de ser creativas. El trabajo de la "co-evolución”, que toma tiempo, permite que la novedad introducida por la aleatoriedad se haga compatible con lo ya existente, y desata cambios ulteriores en lo ya existente, alimentados por la aleatoriedad. En el arte, o bien se crea novedad dentro de un canon artístico establecido, o bien se crean nuevos cánones, transformando los antiguos. La relación entre el blues y el rock es como la relación que hay entre la vejiga natatoria (órgano de flotación de los peces) y el pulmón, que surgió mediante pequeñas y sucesivas modificaciones de la vejiga natatoria (ver Darwin, 2006, p. 120-121). En el arte y la naturaleza es interesante el estudio de las variedades intermedias: por ejemplo Paranoid, de Black Sabbath, un oscuro y maravilloso eslabón perdido entre el blues y el rock.

No es fácil aunar coherencia y diversidad. En nuestros ejemplos se necesita de largos lapsos de tiempo evolutivo, o bien de genio musical, y de mucho trabajo. Es más fácil sacrificar alguno de los dos valores, en cuyo caso suele ser la diversidad la que resulta sacrificada. La música punk (hija del rock, nieta del blues), que, de manera programática, busca ser un vehículo de expresión para quienes no son grandes virtuosos musicales, no es, a pesar de la ideología rebelde que la anima, un derroche de diversidad y capricho. Por el contrario, tiende a seguir una estructura bien definida de coro-verso-coro, a usar pocos instrumentos, acordes y patrones de percusión; el bajo, a diferencia del rock clásico, sigue estrictamente los acordes de la guitarra eléctrica; y la batería evita variar sobre el ritmo básico. No hay solos.

La mera variedad es locura; la mera cohesión es tolerable y accesible a cualquiera. Cualquiera puede hacer o escuchar música punk (es la idea). Hay que aprender a escuchar a Bach (o a Led Zeppelin). Si no somos hábiles para maximizar tanto cohesión como diversidad, si no podemos conservar esta tensión, nos encontramos, por lo general, con la mera cohesión; pequeños pueblos cerrados y conservadores, formas trilladas y repetitivas en los seriados de televisión, círculos sociales cuyo único y alto precio de ingreso es la conformidad a ciertos valores y costumbres. Un ejemplo de exceso en el otro sentido podría ser cierto arte conceptual, que produce piezas chocantes $\mathrm{u}$ ofensivas sin atención alguna al oficio; pero, por lo general, cuando no podemos conciliar diversidad y cohesión preferimos esta última.

\section{Complejidad y poder de obrar}

Comenzamos con dos variables, diversidad y cohesión, anotando que están en tensión. Mostramos que, no obstante, pueden coexistir, siempre y cuando haya un tercer término, un proceso, trabajo u oficio mediante el cual la diversidad de un sistema se integre de manera coherente a una totalidad. A continuación, exploraremos esta misma idea en la filosofía de Spinoza.

Un cuerpo, en Spinoza, es un proceso. Es decir, lo que da identidad a un cuerpo no es el estar compuesto de cierta materia determinada. Los torbellinos, las sociedades, los seres humanos (todos estos “cuerpos" en la ontología de Spinoza) 
son flujos permanentes de materia. Lo que constituye su identidad es ser procesos que conservan una estructura determinada. ¿Cómo se conserva un proceso en el tiempo? Se trata de un proceso que se produce a sí mismo, un proceso cuyo resultado es el proceso mismo. He aquí el conatus, el esfuerzo por perseverar en el ser de cada cosa, que no es nada distinto de la esencia actual de la cosa misma (Spinoza, 1999, 3p4, 3p5, 3p6, 3p7, $3 \mathrm{p} 8$ у $3 \mathrm{p} 9)$.

La ontología de procesos de Spinoza permite pensar en cuerpos compuestos; es decir, en procesos que hacen parte de procesos de orden superior. Mi pulmón es un cuerpo, con su propio conatus, que se esfuerza por perseverar en su ser pulmón. Pero este proceso entronca con el proceso que es mi cuerpo, que intenta también perseverar en su ser; y esta tarea incluye un esfuerzo por conseguir que mis pulmones, mi estómago, etc., perseveren en su ser. Así mismo, las células de mi pulmón son cuerpos, que intentan conservar su integridad estructural mediante membranas semipermeables, y cuyo esfuerzo para perseverar en su ser células es compatible con el esfuerzo del pulmón por perseverar en su ser pulmón y de mi cuerpo de perseverar en su ser cuerpo. De modo que, con Spinoza, podemos pensar no sólo en cuerpos compuestos sino en niveles de observación (recordemos que antes hablábamos del lobo Pedro que, haciéndole un daño al ciervo Juan, beneficia no obstante a la raza de los ciervos): lo que sea una parte y lo que sea el todo depende del nivel de anidamiento, dentro de otros procesos, del proceso que estamos observando.

En un pasaje notable de la Ética, después de la proposición 13 de la segunda parte, Spinoza hace un rápido tránsito de niveles de observación desde los cuerpos más simples hasta la naturaleza entera considerada como un solo cuerpo. A cada paso muestra cómo, a medida que un cuerpo se hace más complejo, puede modificarse de diversas maneras sin dejar de ser lo que es. Un cuerpo muy simple se define por un tipo de movimiento muy simple; cualquier modificación de este movimiento hace que el cuerpo deje de ser lo que es. Un cuerpo se hace complejo a medida que incorpora en su forma cada vez más tipos de movimientos diferentes, y logra armonizarlos con arreglo a una única forma, si bien compleja. Un cuerpo así puede tener muchos estados diferentes sin que dichos cambios de estado constituyan su destrucción. En el límite, tenemos a la naturaleza entera, un cuerpo compuesto de cuerpos compuestos, que unifica un máximo de diversidad:

$[\mathrm{H}]$ asta ahora hemos concebido un individuo que no se compone sino de cuerpos que sólo se distinguen entre sí por el movimiento y el reposo, la rapidez y la lentitud, esto es, que se compone de los cuerpos más simples. Si ahora concebimos otro, compuesto de varios individuos de distinta naturaleza, hallaremos que puede ser afectado de muchas otras maneras conservando, no obstante, su naturaleza. En efecto, supuesto que cada una de sus partes está compuesta de varios cuerpos, cada parte podrá, sin cambio alguno de su naturaleza, moverse más lenta o más rápidamente, $\mathrm{y}$, por consiguiente, comunicar sus movimientos a las otras más aprisa o más despacio. Si concebimos, además, un tercer género de individuos, compuesto de individuos del segundo género, hallaremos que puede ser afectado de otras muchas maneras, sin cambio alguno en su forma. Y si continuamos así hasta el infinito, concebimos, fácilmente, que toda la naturaleza es un solo individuo, cuyas partes - esto es, todos los cuerposvarían de infinitas maneras, sin cambio alguno del individuo total[...] (Spinoza, 1999, 2p13, L7).

Aquí, vemos que el poder de perseverar en el ser de un cuerpo compuesto está aunado a su complejidad. Un cuerpo es poderoso porque puede asumir muchos estados, responder a muchas situaciones; y esta variabilidad de un cuerpo se debe a la multiplicidad de elementos y de relaciones que lo componen. Podemos poner ejemplos: un ecosistema con gran biodiversidad puede sobrevivir a muchas perturbaciones que acabarían con un ecosistema más pobre: la multiplicidad de funciones, nichos y posibilidades de variación del ecosistema diverso le permiten un amplio repertorio de respuestas a variaciones en el clima, en la población de alguna especie determinada, etc. Entre 1845 y 1852 Irlanda perdió casi la cuarta parte de su población, debido al hambre y la emigración; ila causa? La economía agrícola del país estaba basada casi exclusivamente alrededor de la papa de 
modo que ésta se desmoronó con la plaga del tizón tardío, que afectaba a este cultivo. Hay, pues, una relación entre la complejidad de un cuerpo y su poder de obrar: Walt Whitman, hablando de la grandeza de su propia alma, escribió así "¿Y si me contradigo? Pues bien, me contradigo. Soy grande, contengo multitudes" (Whitman, 2004, pp. 1325-1327). ${ }^{2}$

Un cuerpo es complejo porque está compuesto de múltiples cuerpos, a su vez compuestos. Cada cuerpo, a cualquier nivel de composición, "se esfuerza, cuanto está a su alcance, por perseverar en su ser" (Spinoza, 1999, 3p6), pues es, esencialmente un determinado proceso que expresa su propio poder de obrar, de modo que "El esfuerzo con que cada cosa intenta perseverar en su ser no es nada distinto de la esencia actual de la cosa misma" (Spinoza, 1999, 3p7). ¿Cómo es posible un cuerpo compuesto? Un cuerpo puede hacerse complejo y diverso siempre y cuando pueda constituirse en un proceso tal que los diferentes cuerpos que lo componen puedan realizar su conatus, perseverar en su ser, como partes de un proceso de orden superior. En una relación genuinamente amorosa, cada miembro es genuinamente sí mismo, persevera en su ser, gracias a la relación amorosa. En un ecosistema, cada cosa es lo que es, el tigre "tigrea", las abejas "abejean", las flores "florean", sólo gracias al ecosistema, a la red de relaciones que permite que cada miembro persevere en su ser.

Lograr la composición implica, pues, lo siguiente: a) poner a los diversos entes en un determinado nivel de observación en relaciones de simbiosis, b) poner en relación de simbiosis los diversos niveles de composición de un sistema complejo, y c) conciliar la actividad mediante la cual las partes de un sistema perseveran en su ser con la actividad de perseverar en el ser del sistema en su totalidad. La composición no se consigue cuando el todo se traga y homogeniza sus partes componentes (el resultado de esto es un cuerpo bien simple y poco flexible, como lo fue, por ejemplo, la Unión Soviética), sino cuando el todo busca una forma capaz de permitir que cada una de sus

2 Todas las citas de Whitman traducidas del inglés al español por el autor. partes persevere en su ser. Una manera de visualizar esto es comparando una crema con un sancocho. En la crema, los diferentes elementos son homogeneizados mediante una licuadora; en el sancocho, si bien hay un plato de orden superior llamado sancocho con un sabor reconocible, al mismo tiempo los diferentes ingredientes se distinguen entre sí y conservan su singularidad.

\section{Diversidad, cohesión y tensión}

E pluribus unum: el sello de los Estados Unidos propone como aspiración histórica que de los muchos se haga uno. En realidad hay dos maneras de cumplir esta misión: lo uno puede ser complejo, y contener lo múltiple; o bien puede ser un uno homogéneo, producto del poder coactivo, la servidumbre y la imitación.

Etienne de la Boétie, a mediados del siglo XVI, comprendió muy bien los mecanismos mediante los cuales el tirano pone bajo su yugo a un pueblo: la costumbre, la superstición y la estupidez (que el tirano se esfuerza por fomentar en su pueblo) pero, sobre todo, la voluntad de dominación, que hace que los hombres acepten su servidumbre a cambio de dominar a otros, formando una cadena de sumisión:

Ni la caballería ni la infantería constituyen la defensa del tirano[... Son cuatro o cinco los que sostienen al tirano, cuatro o cinco los que imponen por él la servidumbre en toda la nación [...] compañeros de sus placeres, rufianes de sus voluptuosidades, y los que se reparten el botín de sus pillajes [...] Estos [...] tienen a seiscientos hombres bajo su poder, a los que manipulan y a quienes corrompen como han corrompido al tirano. Estos seiscientos tienen bajo su poder a seis mil, a quienes sitúan en cargos de cierta importancia, a quienes otorgan el gobierno de las provincias, o la administración del tesoro público, con el fin de favorecer su avaricia y su crueldad, de ponerla en práctica cuando convenga y de causar tantos males por todas partes que no puedan mover un dedo sin consultarlos, ni eludir las leyes y sus consecuencias sin recurrir a ellos. Extensa es la serie de aquéllos que siguen a éstos. El que quiera entretenerse devanando esta red, verá que no son seis mil, sino cien mil, millones, los que tienen sujeto al tirano y los que conforman entre ellos una cadena ininterrumpida que se remonta hasta él (De la Boetie, 2009, pp. 67-68). 
Cada esbirro se somete a su jefe para poder hacerse jefe a su vez de otros tantos esbirros; cada esclavo se somete a un amo para poder tener esclavos a su vez. ¿La constante en esta cadena de tiranías? Cada eslabón debe ceder su libertad, hacerse menos de lo que es, esclavizarse. De este modo se consigue, sin duda, la cohesión, pero al precio de la diversidad. El resultado no es un cuerpo compuesto y complejo, sino una masa homogénea, hecha de partes indistintas.

E pluribus unum: hay quienes celebran la actual homogeneización del mundo que encabeza Estados Unidos. Thomas Friedman celebra la nueva pax americana: no hay guerra entre dos países que tengan McDonald's, pues están, más bien, dedicados a beber las mieles de la globalización. La pérdida de libertad que implica la aceptación de las políticas neoliberales (precio de entrada al comercio global), la celebra como una camisa de fuerza dorada. Francis Fukuyama, por su parte, se erigió en profeta del fin de la historia (quizás viva para ver refutada su profecía). De lo múltiple se da lo uno, pero a expensas de la multiplicidad.

Cohesión sin diversidad: no es esto lo que buscan los cuerpos compuestos que aumentan su poder al tiempo que aumentan su complejidad. En Spinoza, el poder de un cuerpo es la misma capacidad de ser afectado de muchísimas maneras, esto es, de ser múltiple:

Aquello que propicia que el cuerpo humano sea afectado de muchísimos modos, o aquello que lo hace apto para afectar de muchísimos modos los cuerpos exteriores, es útil al hombre, y tanto más útil cuanto más apto hace al cuerpo para ser afectado, o para afectar a otros cuerpos, de muchísimas maneras; $y$, por contra, es nocivo lo que hace al cuerpo menos apto para ello (Spinoza, 1999, 4p38).

En Salut au monde (saludo al mundo; o bien, salud al mundo), Whitman celebra su propia capacidad (y la capacidad de su patria, o de la patria idealizada que habita sus poemas) de ser múltiple, de acoger dentro de su unidad la multiplicidad, de ser afectado de muchas maneras. Esta capacidad tiene que ver con la de escuchar:
Escucho al trabajador cantando, y a la esposa del granjero cantando,

escucho en la distancia los sonidos de los niños y los animales

temprano en el día,

Escucho los gritos gregarios de australianos a la caza del caballo salvaje,

Escucho a españoles bailar con castańetas a la sombra del castaño, al ritmo del rabel y la guitarra

Escucho los ecos continuos del Támesis

Escucho las bravas canciones francesas de libertad

$[\ldots]$

Escucho al Hindú, enseñando a su alumno favorito los amores, guerras, adagios, transmitidos con seguridad hasta este día desde los poetas que escribieron hace tres mil años. (Whitman 2004, pp. 24-43).

A lo largo de su poesía, vemos a Whitman celebrar su capacidad de ser múltiple, de contener o devenir múltiples maneras de ser:

Soy de viejos y jóvenes, lo mismo de necios que de sabios[...]

Tan norteño como sureño, un granjero tranquilo y hospitalario,

habito las orillas del Oconee.

Un yanqui en mi camino aprestándome para el comercio[...]

Uno de Kentucky caminado el valle de Elkhorn en mis botas de piel de venado, uno nativo de Luisiana o de Georgia[...]

Camarada de barqueros y carboneros, camarada de todos los que dan la mano y la bienvenida para beber y comer carne[...]

De cada color y casta soy, de cada rango y religión

Granjero, mecánico, artista, caballero, marino, cuáquero,

Prisionero, diletante, revoltoso, abogado, médico, sacerdote (Whitman, 2004, pp. 330-348).

¿Cómo conserva Whitman su unicidad, su esencia propia, al tiempo que abarca con su ser a todo el universo? ¿Cómo persevera en su ser al tiempo que es afectado de muchísimas maneras? Veamos las dos siguientes líneas del poema: 
Todo lo resisto mejor que mi propia diversidad y respiro el aire, pero dejo bastante tras de mí (Whitman, 2004, pp. 349-350).

Los diferentes afectos no son fuente de tensión, lo es la propia diversidad del poeta. La diversidad es fuente y producto de esfuerzos; respirar el aire dejando suficiente para todo lo demás es un emprendimiento. E pluribus unum: el uno de Whitman, el uno de los Estados Unidos cantados y soñados por Whitman, es uno en tensión, en permanente esfuerzo por dar cabida a cada elemento (poéticamente, es el esfuerzo y el arte mediante el cual las enumeraciones de Whitman resultan vívidas y estimulantes, en lugar de tediosas). Es uno en tensión: no pertenece a Whitman la pétrea pax americana. En lugar de ello hay un combate amoroso, o una violenta copulación (consensual, pero de uñas en la espalda y sangre en los labios), una tensión permanente en la integración de lo múltiple en unidades dinámicas, permanentemente negociadas. La tensión es el precio de la democracia a la que canta Whitman, es la forma en que pueden contenerse multitudes.

\section{Diversidad y tolerancia}

Frente a la diversidad, sea racial, cultural o sexual, se habla muchas veces de tolerancia. Cuando un radio no tiene muy buena señal, toleramos el ruido de fondo. Cuando el anjeo se rompe, hemos de tolerar a los mosquitos. La tolerancia no pide sino un mínimo de aceptación de la diversidad: "la acepto porque no es de mi incumbencia, la acepto siempre y cuando no me incumba". Si bien este mínimo es mejor que la intolerancia, resulta que de la mera tolerancia no resultan sociedades diversas. Por el contrario, resultan sociedades insulares, colecciones de ghettos yuxtapuestos que procuran no tocarse, en aras de la tolerancia.

Hoy en día escuchamos cosas así: "No tengo ningún problema con los homosexuales, siempre y cuando no se besen en la calle". Esto es un pensamiento "tolerante". Este tipo de discriminación suave podría combatirse desde las ideas de justicia y reciprocidad. Basta con cambiar un término en la frase: "No tengo ningún problema con los heterosexuales, siempre y cuando no se besen en la calle”. ¿Qué tal suena? Este tipo de argumentos son válidos; después de todo, la paridad y la reciprocidad son la base del pensamiento ético. No obstante, no es por medio de este tipo de argumentos que se pasa de una sociedad insular y fragmentada, cohesionada sólo en sus diversas islas, a una sociedad verdaderamente diversa, en la que las variables en tensión, cohesión y diversidad, tienden ambas a maximizarse. Por supuesto, conseguir la verdadera diversidad es más difícil que conseguir la tolerancia. Como es más difícil componer música barroca que reggaeton, o conseguir un ecosistema maduro que sembrar un monocultivo de papa. Izquierda o derecha, diversidad o cohesión. Hay una tercera alternativa, hacia delante, hacia la complejidad, hacia hacernos más de lo que somos.

\section{Referencias Bibliográficas}

Bateson, G. (1993). Espiritu y naturaleza. Buenos Aires: Amorrortu.

De la Boétie, É. (2009). Discurso sobre la servidumbre voluntaria. La Plata: Derramar.

Darwin, C. (2006). On the origin of the species. Nueva York: Dover.

Leibniz, G. W. (1984). Monadología. Barcelona: Orbis.

Spinoza, B. (1999). Ética. Madrid: Alianza.

Wilson, E. O. (1984). Biophilia. Londres: Harvard University Press.

Whitman, W. (2004). The Complete Poems. Londres: Penguin. 